\title{
PERTURBATION OF $(m, p)$-ISOMETRIES BY NILPOTENT OPERATORS AND THEIR SUPERCYCLICITY
}

\author{
Masoumeh FAghiH-Ahmadi, SAEEd YARMAhmoodi \\ AND KARIM HEDAYATIAN
}

Abstract. A bounded linear operator $T$ on a Hilbert space $H$ is an $(m, p)$-isometry if

$$
\sum_{k=0}^{m}(-1)^{k}\left(\begin{array}{c}
m \\
k
\end{array}\right)\left\|T^{k} x\right\|^{p}=0
$$

for all $x \in H$, in which $p \in[1, \infty)$ and $m \geqslant 1$. In this paper, two significant results will be proved. First, we introduce some perturbations of $(m, p)$-isometries which are $(n, p)$-isometries for some suitable $n$. Indeed, we show that the sum of an $(m, p)$-isometry and a commuting nilpotent operator of degree $r$ is a $(p r-p+m, p)$-isometry for every even number $p$. As an application, the second result is to prove that such operators are not $N$-supercyclic for any positive integer $N$, even if $p$ is a rational number. These results generalize the previous works on $m$-isometries.

Mathematics subject classification (2010): 47B99.

Keywords and phrases: Isometry, $m$-isometry, nilpotent operator, $N$-supercyclic operator.

\section{REFERENCES}

[1] J. Alger, M. Stankus, $m$-isometric transformations of Hilbert space I, Integr. Equ. Oper. Theory 21 (1995), 383-429.

[2] J. AgLeR, M. StankUs, $m$-isometric transformations of Hilbert space II, Integr. Equ. Oper. Theory 23 (1995), 1-48.

[3] J. AgleR, M. StanKus, m-isometric transformations of Hilbert space III, Integr. Equ. Oper. Theory 24 (1996), 379-421.

[4] S. I. Ansari, P. S. Bourdon, Some properties of cyclic operators, Acta Sci. Math. (Szeged), 63 (1997), 195-207.

[5] A. Athavale, Some operator theoretic calculus for positive definite kernels, Proc. Amer. Math. Soc. 112 (3) (1991), 701-708.

[6] F. BAYART, m-isometries on Banach spaces, Math. Nachr. 284 (2011), 2141-2147.

[7] F. Bayart, E. Matheron, Dynamics of Linear Operators, vol. 179, Cambridge University Press, 2009.

[8] T. Bermúdez, I. Marrero, A. Marinón, On the orbit of an $m$-isometry, Integr. Equ. Oper. Theory 64 (4) (2009), 487-494.

[9] T. BERMúdeZ, A. MARTINón, E. Negrin, Weighted shift operators which are $m$-isometries, Integr. Equ. Oper. Theory 68 (3) (2010), 301-312.

[10] T. Bermúdez, A. Martinón, V. Müller and J. A. Noda, Perturbation of $m$-isometries by nilpotent operators, Abstr. Appl. Anal. 2014 (2014), Article ID 745479, 6 pages.

[11] T. BERMÚdeZ, A. MARTINÓN AND J. A. NODA, An isometry plus a nilpotent operator is an misometry, Applications, J. Math. Anal. Appl. 407 (2) (2013), 505-512.

[12] P. S. Bourdon, N. Feldman, J. Shapiro, Some properties of $N$-supercyclic operators, Studia Math. 165 (2) (2004), 135-157. 
[13] M. Faghin-Ahmadi, Powers of A-m-isometric operators and their supercyclicity, Bull. Malays. Math. Sci. Soc. 39 (3) (2016), 901-911.

[14] M. FAGHIH-AhmADI, K. HedAyATIAn, Hypercyclicity and supercyclicity of $m$-isometric operators, Rocky Mountain J. Math., 42 (1) (2012), 15-23.

[15] N. Feldman, $n$-supercyclic operators, Studia Math. 151 (2) (2002), 141-159.

[16] K. G. Grosse-Erdmann, A. Peris Manguillot, Linear Chaos, Springer-Verlag London Limited, 2011.

[17] G. Gu And M. Stankus, Some results on higher order isometries and symmetries: products and sums with a nilpotent operator, Linear Algebra Appl. 469 (2015), 500-509.

[18] H. M. Hilden, L. J. Wallen, Some cyclic and non-cyclic vectors of certain operators, Indiana Univ. Math. J. 23 (1974), 557-565.

[19] P. Hoffmann, M. Mackey And M. Searcóid, On the second parameter of an $(m, p)$-isometry, Integr. Equ. Oper. Theory 71 (2011), 389-405.

[20] T. LE, Algebraic properties of operator roots of polynomials, J. Math. Anal. Appl. 421 (2) (2015), $1238-1246$.

[21] V. G. MILLER, Remarks on finitely hypercylic and finitely supercyclic operators, Integr. Equ. Oper. Theory 29 (1997), 110-115.

[22] O. A. M. Sid Ahmed, m-isometric operators on Banach spaces, Asian-European J. Math. 3 (1) (2010), 1-19.

[23] S. Yarmahmoodi, K. Hedayatian, B. Yousefi, Supercyclicity and hypercyclicity of an isometry plus a nilpotent, Abstr. Appl. Anal. 2011 (2011), Article ID 686832, 11 pages. 